\title{
Study on Kerosene-submerged Jet Electrochemical Machining and Optimization of the Electrochemical Machining Parameters
}

\author{
Xinchao Li, Pingmei Ming ", Xinmin Zhang*, Shen Niu, Xingshuai Zheng, Liang Yan, Wei Wang, \\ Yunyan Zhang \\ Institute of Non-traditional Machining \& Equipment, Henan Polytechnic University, Jiaozuo, 454000, \\ China \\ *E-mail: mingpingmei@163.com, hpuzxm@163.com
}

doi: $10.20964 / 2021.01 .51$

Received: 24 September 2020 / Accepted: 9 November 2020 / Published: 30 November 2020

\begin{abstract}
Kerosene-submerged jet electrochemical machining (Jet-ECM) has been proven to be an effective process to enhance the machining localization of Jet-ECM. However, this process can only show its best capabilities and advantages under appropriate electrochemical machining parameters. Its machining effects will be weakened when the workpiece surface is relatively large or electrolytic products generated during machining are quite more, which may cause the electrolyte and electrolytic products to accumulate on the workpiece surface, thereby affecting its mass transfer environment. Therefore, for further improving its process capabilities, it is necessary to eliminate the accumulation of electrolyte and electrolytic products on the workpiece surface. And thus, this paper focuses on optimizing its electrochemical machining parameters and investigating the surface effect and shape effect of the workpiece on its machining localization and machining accuracy experimentally and theoretically. It was found that the surface effect and shape effect of the workpiece has a significant influence on its machining localization and machining accuracy. And kerosene medium instead of air medium surrounding the electrolyte-jet can improve the machining localization of Jet-ECM. Besides, compared with traditional Jet-ECM, kerosene-submerged Jet-ECM can produce high accuracy microstructure with a smooth surface. Additionally, micro-sized features fabricated by Jet-ECM on the small surface flat workpiece and curved surface workpiece feature higher machining accuracy and better surface quality than the large surface flat workpiece.
\end{abstract}

Keywords: Kerosene-submerged jet electrochemical machining; machining localization; machining accuracy; surface effect; shape effect

\section{FULL TEXT}

(C) 2021 The Authors. Published by ESG (www.electrochemsci.org). This article is an open access article distributed under the terms and conditions of the Creative Commons Attribution license (http://creativecommons.org/licenses/by/4.0/). 